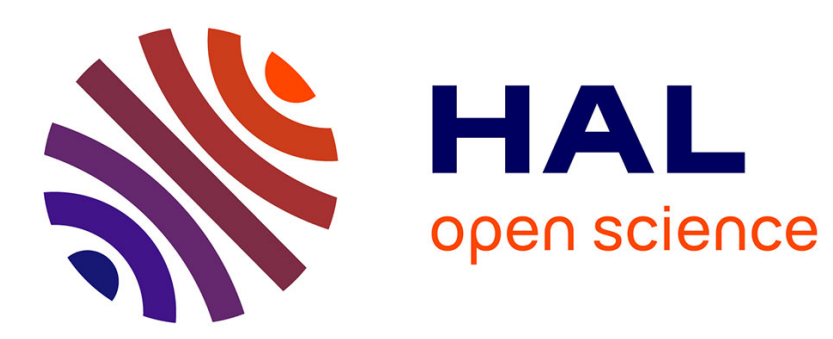

\title{
La parataxe asyndétique dans les contes de La Maison Tellier de Maupassant
}

\author{
Véronique Magri-Mourgues
}

\section{To cite this version:}

Véronique Magri-Mourgues. La parataxe asyndétique dans les contes de La Maison Tellier de Maupassant. L'information grammaticale, 2011, 131, pp.23-27. hal-01226790

\section{HAL Id: hal-01226790 \\ https://hal.science/hal-01226790}

Submitted on 10 Nov 2015

HAL is a multi-disciplinary open access archive for the deposit and dissemination of scientific research documents, whether they are published or not. The documents may come from teaching and research institutions in France or abroad, or from public or private research centers.
L'archive ouverte pluridisciplinaire HAL, est destinée au dépôt et à la diffusion de documents scientifiques de niveau recherche, publiés ou non, émanant des établissements d'enseignement et de recherche français ou étrangers, des laboratoires publics ou privés. 


\title{
La parataxe asyndétique \\ dans les contes de La Maison Tellier ${ }^{l}$ de Maupassant
}

\author{
Véronique Magri-Mourgues \\ Université Nice Sophia-Antipolis \\ UMR 7320 Bases, Corpus, Langage
}

Pour assurer une assise théorique sinon consensuelle, du moins susceptible d'offrir une certaine stabilité, j'adopterai le point de vue de la plupart des grammairiens pour définir ce qu'il faut entendre par «parataxe asyndétique », terme double qui permet d'envisager le binôme parataxe / asyndète.

La parataxe est un mode de construction reliant des unités autonomes et non hiérarchiquement ordonnées, qui s'oppose à l'hypotaxe. Elle comprend alors juxtaposition et coordination ou, pour donner une description plus homogène des faits organisée autour du principe de coordination, elle se décline en un mode de coordination explicité par la présence d'un mot coordonnant et un mode de coordination à ligateur zéro - la juxtaposition ou asyndète.

La parataxe asyndétique ainsi définie ouvre la voie à des approches situées à différents niveaux mais complémentaires. Elle est un procédé syntaxique qui inscrit les unités juxtaposées dans une séquentialité a priori non hiérarchisante, présentées comme autonomes mais dont l'insertion même dans la chaîne du discours problématise la relation. L'empan de l'asyndète est un premier paramètre : la juxtaposition peut concerner les phrases, les sousphrases, les syntagmes, les mots. Des questions se posent qui concernent la relation éventuellement instaurée entre les unités. La succession d'unités doit-elle être appréhendée en termes de discontinuité formelle voire de discordance sémantique, ou bien quelle que soit l'échelle où s'exerce la juxtaposition, une relation d'ordre logique peut-elle être reconstruite entre les unités ou, même, un rapport sémantique est-il susceptible d'être déduit de leur succession? Une valeur sémantique est-elle à inférer de l'ordonnancement des unités ? Sur le plan stylistique, il faut se demander quel peut être l'effet produit par la succession d'unités simplement juxtaposées; par conséquent, dépasser l'observation linguistique microstructurale pour atteindre le niveau de l'analyse globale du texte.

Dans le cas des Contes de La Maison Tellier, c'est la récurrence de ce phénomène simplement observée qui attire d'abord l'attention et qui pose la question au stylisticien de la possible conversion de la répétition en itération signifiante. L'aller-retour de la forme globale de la nouvelle aux micro-structures s'impose alors. Pourrait-on, à partir de l'analyse d'une construction syntaxique récurrente, extrapoler une amorce d'interprétation qui porterait sur le genre même de la nouvelle ? Une équivalence linguistique des propriétés de la nouvelle pourrait-elle être ici trouvée, comme une réalisation prototypique concrète des constantes génériques définitoires de ce type de texte ?

\section{Les dispositifs en série - analyse microstructurale}

Afin de délimiter le corpus d'étude, on retiendra que les cas de parataxe asyndétique sont ceux où il est grammaticalement possible d'ajouter un coordonnant. Ce critère permet d'exclure les cas où une relation attributive associe les termes reliés, comme dans les exemples suivants avec des variantes :

\footnotetext{
${ }^{1}$ Maupassant, La Maison Tellier[1881]. Paris, Folio classique, (1973 et 1995). Le corpus d'étude comprend les nouvelles: Sur l'eau, Histoire d'une fille de ferme, En famille, Le Papa de Simon, Une partie de campagne, Au printemps, La Femme de Paul.
} 
[1] Je fis, au bout de quelques jours, la connaissance d'un de nos voisins, un homme de trente ou quarante ans. (Sur l'eau, 72)

[2] C'est en effet le plus sinistre des cimetières, celui où l'on n'a point de tombeau. (Sur l'eau, 73)

[3] Chose étrange, je n'avais plus peur. (Sur l'eau, 78)

Une relation d'identification est à reconstruire entre les termes associés dans une relation strictement binaire; la relation est de type appositif dans ces trois énoncés, à support propositionnel en [3]. Des différences qui se situent aux niveaux stylistique et informationnel distinguent simplement ces trois exemples. Le procédé d'identification repose sur la construction emphatique par le présentatif «c'est» dans l'exemple [2] tandis que l'exemple [3] inverse l'ordre attendu thème-prédicat en faveur de la mise en valeur du prédicat.

Cette condition exclut encore les cas de structure à rallonge où un élément vient compléter le syntagme précédent.

[4] Quand la fille eut fini sa besogne, essuyé la table, nettoyé la cheminée et rangé les assiettes sur le haut dressoir au fond près de l'horloge en bois au tictac sonore [...]. (Histoire d'une fille de ferme, 79)

L'exemple [4] présente cette particularité d'une phrase qui juxtapose, dans sa première partie, des participes passés dépendants du même auxiliaire «eut» et, dans sa seconde partie, une structure à différencier de la structure asyndétique; «au fond», «près de l'horloge » s'agrègent à «haut dressoir»; « en bois », «tictac sonore » caractérisent « horloge » dans une structure de juxtaposition non coordonnante, ou « maillage $»^{2}$.

Dans les exemples où un mot coordonnant peut être inséré, c'est l'empan de la juxtaposition qui peut servir de premier critère typologique.

\subsection{L’empan de la liste asyndétique}

Les séquences à vocation descriptive privilégient les dispositifs syntaxiques sériels, et ceci à plusieurs niveaux de la phrase. Des phrases qu'une ponctuation forte délimite sont juxtaposées ; une galerie de portraits se déploie :

[5] M. Dufour, que secouait un hoquet violent, avait déboutonné son gilet et le haut de son pantalon tandis que sa femme, prise de suffocations, dégrafait sa robe peu à peu. L'apprenti balançait d'un air gai sa tignasse de lin et se versait à boire coup sur coup. La grand-mère, se sentant grise, se tenait fort raide et fort digne. Quant à la jeune fille, elle ne laissait rien paraître, son œil s'allumait vaguement, et sa peau très brune se colorait aux joues d'une teinte plus rose. (Une partie de campagne, 149)

Des sous-phrases sont juxtaposées; les segments sont alors séparés par une ponctuation faible.

[6] Les serins accrochés aux fenêtres s'égosillaient; les bonnes chantaient à tous les étages ; une rumeur gaie montait de la rue ; et je sortis, l'esprit en fête, pour aller je ne sais où. (Au printemps, 156)

[7] L'eau clapotait lugubrement, le vent soufflait, il faisait froid, l'obscurité était profonde. (Sur l'eau, 78)

Des structures prédicatives sont juxtaposées enfin, qu'il s'agisse de syntagmes nominaux, d'adjectifs ou de verbes. Syntaxiquement, la mise en facteur commun d'un élément régisseur autorise la construction en chaînes d'éléments, dans des structures qui reposent sur l'enchâssement quelquefois :

\footnotetext{
${ }^{2}$ M. Wilmet (1997). § 234 et 708 .
} 
[8] Alors, peu à peu, elle accapara la besogne autour d'elle, fit renvoyer une servante qui devenait inutile [...], économisa sur le pain, sur l'huile et sur la chandelle, sur le grain qu'on jetait trop largement aux poules, sur le fourrage des bestiaux qu' on gaspillait un peu. (Histoire d'une fille de ferme, 87)

Cet exemple joue d'abord sur la mise en facteur commun du sujet «elle » qui régit trois prédicats verbaux; le dernier d'entre eux exerce ensuite son rôle recteur sur quatre compléments juxtaposés et symétriquement introduits par la préposition «sur». Si la succession paraît ainsi soigneusement ordonnée et maîtrisée dans cet exemple, l'homogénéité syntaxique entre les éléments juxtaposés n'est cependant pas toujours assurée comme dans l'exemple suivant qui associe quatre constructions différentes :

[9] L'ayant vue s'étendre à l'ombre, il était venu, à pas de loup, retenant son haleine, les yeux brillants, avec des brins de paille dans les cheveux. (Histoire d'une fille de ferme, 81)

Sur le plan syntaxique, cet exemple de juxtaposition peut paraître quelque peu problématique en raison même de l'hétérogénéité catégorielle de ses constituants pouvant induire une incidence différente d'un élément à l'autre; la discordance introduite par une éventuelle variation d'incidence freinerait alors l'interprétation par la coordination syntaxique implicite. « À pas de loup », qui s'assimile à un groupe adverbial, paraît devoir modifier naturellement le verbe ; le syntagme «avec des brins de paille dans les cheveux » peut recevoir la même analyse, sauf à éluder la préposition « avec », tandis que les deux autres groupes fonctionnent davantage, en raison de leur nature, comme appositions au terme support, le pronom «il». Quoi qu'il en soit, la mise en série de ces différentes unités dans la phrase nivelle leurs discordances et simule une équivalence syntaxique entre elles; la conjonction « et » pourrait sans peine être rajoutée entre les deux premières unités par exemple. Dans un énoncé de ce type, aucun indice explicite de structuration interne n'est décelable mais, par-delà l'hétérogénéité syntaxique, c'est bien sûr l'unité descriptive d'un même personnage qui assure la cohérence sémantique de l'énumération, en pointant des détails signifiants : le silence qui explique la surprise de Rose la servante, le désir du personnage lisible dans ses yeux brillants, son côté rustre aussi révélé par les brins de paille dans les cheveux.

\subsection{La structure interne de la liste}

Quand on a affaire à une liste qui repose sur une symétrie logique, celle-ci impose son ordonnancement attendu et appelle la clôture comme le balancement «le jour, la nuit »

[9] Elle ne dormait plus la nuit ; elle y pensait tout le jour. (Histoire d'une fille de ferme, 87)

Ou encore avec des adverbes temporels qui se répondent et assènent un jugement sans retour :

[10] Elle n'avait jamais été jolie ; elle était laide maintenant, de petite taille et maigrelette. (En famille, 107)

Dans tous les autres cas se pose la question de la structure interne de la liste et donc de la succession non aléatoire des éléments juxtaposés et aussi de sa clôture. Une liste ouverte, qui se constitue par empilement d'items dont aucune finalité affirmée ne dicte l'agencement, pourrait théoriquement se dérouler à l'infini.

Des indices compensatoires assurent une structuration de la liste. Ce peut être la reprise anaphorique d'un mot à l'initiale de chaque segment qui devient comme une mesure rythmant la phrase qu'on pourrait dire à étagement ou tabulaire. L'exemple [8] qui reprend en parallèle 
la préposition «sur » en est un exemple parmi tant d'autres. L'anaphore au sens rhétorique du terme fonctionne comme "outil coordinatif de remplacement qui laisse subsister et même souligne la juxtaposition $»^{3}$. La reprise anaphorique de l'adverbe relatif « où » est manifeste encore dans cet exemple.

[11] Pour lui, c'est la chose mystérieuse, profonde, inconnue, le pays des mirages et des fantasmagories, où l'on voit, la nuit, des choses qui ne sont pas, où l'on entend des bruits que l'on ne connaît point, où l'on tremble sans savoir pourquoi, comme en traversant un cimetière. (Sur l'eau, 72)

Parallèlement à l'anaphore, l'homéotéleute s'exerce à la faveur d'une forme grammaticale constante dans les éléments juxtaposés; c'est aussi un motif euphonique et rythmique de structuration interne de la liste.

[12] Chaque fois qu'on la surprenait cirant, brossant, astiquant ou lessivant. (En famille, 107)

Dans les exemples cités supra [4] et [5], c'est la conjonction « et » ou encore la formule « quant à » qui prennent une valeur conclusive pour fonctionner comme indices de clôture.

Un simple changement de tiroir verbal suffit à assurer la clôture par le contraste qu'il instaure dans l'exemple [13] :

[13] Sa tête se perdait, les cauchemars se succédaient, sa chandelle s'éteignit. (Histoire d'une fille de ferme, 91)

De nombreux exemples de juxtaposition ne présentent aucun signe de clôture :

[14] Une mollesse passait dans le sommeil de la terre, et Caravan buvait cette douceur de la nuit ; il respirait longuement, croyait sentir pénétrer jusqu'à l'extrémité de ses membres une fraîcheur, un calme, une consolation surhumaine. (En famille, 117)

Le jeu sur les formes syntaxiques, qui croisent des paramètres euphoniques quelquefois, assure une cohésion formelle à la juxtaposition ; la cohérence sémantique entre ces éléments est d'un autre ordre et reste indépendante de la structure formelle. Le désordre syntaxique constaté dans certains exemples où aucun indice de structuration n'apparaît ne s'accompagne nullement d'incohérence :

Tout se passe comme si la simple contiguïté [...] constituait à elle seule l'élément du plan de l'expression recouvrant et signalant la relation entre deux unités phrastiques, et fondant ainsi leur succession en signification. ${ }^{4}$

La succession sans coordonnant susceptible d'introduire une relation logique entre les unités ne peut être interprétée en termes de rupture, comme absence d'organisation ou indice de désordre ou de discontinuité. L'absence de ligateur est contrebalancée par un principe d'isotopie qui assure la cohésion sémantique et référentielle ; l'exemple [7] construit une scène à l'atmosphère inquiétante. La juxtaposition de prédicats dont l'enchaînement esquisse cependant une gradation entre des éléments communément admis comme dysphoriques (le vent, le froid, l'obscurité) permet une discrète remotivation de la métaphore qui associe l'adjectif «profonde » au nom « obscurité » par échange allusif d'un prédicat-type de l'eau.

La relation instituée entre les termes juxtaposés est d'ordre discursif et non inscrite en langue. Elle est ainsi susceptible de recouvrir différentes formes, dont celle de la reformulation ${ }^{5}$.

\footnotetext{
${ }^{3}$ G. Antoine, La Coordination, p. 1291, cité par B. Dupriez, Gradus, Paris, U.G.E., 1984, p. 46.

4 A.-J. Greimas (1976), p. 28-29.
} 


\subsection{La reformulation}

Ce processus est défini comme «la variante paraphrastique d'un segment linguistique ». ${ }^{6}$ Pour le dire trivialement, il s'agit d'exprimer de manière différente le même contenu. La reformulation voisine avec la reprise interprétative exprimée par un marqueur comme «c'està-dire $»^{7}$ :

[15] Un rossignol ! c'est-à-dire l'invisible témoin des rendez-vous d'amour qu'invoquait Juliette sur son balcon ; cette musique du ciel accordée aux baisers des hommes ; cet éternel inspirateur de toutes les romances langoureuses qui ouvrent un idéal bleu aux pauvres petits cœurs des fillettes attendries ! (Une partie de campagne, 151)

Le rossignol sert, dans la suite de l'histoire, de relais descriptif pour des réalités bien plus prosaïques que l'univers romantique qui se déplie au gré des trois équivalents nominaux donnés comme explicitation; la reformulation, dans ce cas, prend une tonalité ironique. L'asyndète, qui s'ouvre à droite du marqueur, contraste par sa longueur avec la brièveté du membre explicité ; l'accumulation devient excessive et sert les intentions du narrateur. Cet exemple atteste de deux points essentiels: d'une part, le changement de perspective énonciative articulé par le marqueur «c'est-à-dire » incluant le verbe « dire », originellement de parole et, d'autre part, le fait que la reformulation ne se confond pas avec la répétition pure et simple. Dire autrement, c'est dire autre chose et c'est aussi contribuer à maintenir la cohésion textuelle et à construire la textualité :

La textualité peut être définie comme un équilibre délicat entre une continuité-répétition, d'une part, et une progression de l'information d'autre part. ${ }^{8}$

En l'absence de marqueur comme «c'est-à-dire», la relation entre des unités syntagmatiques est plus problématique. Comment s'organise la succession linéaire des éléments? En supposant que l'ordre des unités n'est pas aléatoire mais dépend d'une intention du narrateur et, quoi qu'il en soit, acquiert un sens par son existence même dans l'espace de la phrase, on peut s'interroger sur les relations sémantiques entre ces unités. La séquence laisse pressentir une progression du premier élément au suivant, jusqu'au dernier qui oriente, par sa place et rétroactivement, le déroulement de l'énoncé dont il est l'aboutissement phrastique.

Une gradation ordonne les unités. Cela est clair avec l'exemple suivant où le dernier prédicat de la première phrase, un nom de valeur adjectivale, est introduit par l'adverbe « presque ». Les mots juxtaposés s'insèrent dans un processus graduel.

[16] Voilà immédiatement mon bonhomme qui s'anime, se transfigure, devient éloquent, presque poète. Il avait dans le cœur une grande passion, une passion dévorante, irrésistible : la rivière. (Sur l'eau, 72)

«Presque » instaure une tension vers un sémème non complètement réalisé en discours et qui marque une progression depuis l'adjectif «éloquente» qui précède et qui se singularise

\footnotetext{
${ }^{5}$ Voir A. Vial (1954), p. 601. L' « essayage » de termes, qui consiste en une « juxtaposition de reprises », est envisagé comme une caractéristique de l'écriture-artiste.

${ }^{6}$ F. Neveu (2004). Dictionnaire des sciences du langage, Paris, Colin, p. 251.

7 Cette expression est analysée tantôt comme locution adverbiale, tantôt comme locution conjonctive de coordination, voire de subordination. Voir A. Steuckardt et A. Niklas-Salminen (2005), p. 256.

8 J.-M. Adam (1990), p. 45.
} 
comme une des caractéristiques de $"$ poète $»^{9}$. Par analogie avec cette construction, on peut inférer qu'une relation graduelle lie les unités qui se succèdent en asyndète. Ce n'est pas une relation synonymique qui associe les verbes «s'anime» et «se transfigure » mais c'est une relation scalaire qui se construit entre eux par le seul fait de la juxtaposition.

Cet exemple joue encore sur la variation des adjectifs avec reprise ou non du substantif support dans la seconde phrase. On peut lire une gradation de cadence majeure entre « grande, dévorante, irrésistible » dont la compatibilité sémantique reste assurée par le partage de sèmes et qui se distinguent par des sèmes particuliers. "Grande passion » reste un syntagme usuel, quasi-galvaudé ; «dévorante » joue sur un emploi métaphorique et «irrésistible » fait passer d'une visée subjectivante à une visée objectivante.

Les exemples de ce type pourraient être multipliés :

[17] Alors commença le délire, ce délire fuyant des gens de la campagne qui se croient frappés par un sort, un besoin fou de partir, de s'échapper, de courir devant le malheur comme un vaisseau devant la tempête. [...]

Ce serait fini de souffrir là-dedans, fini pour toujours. Elle ne pensait plus à son enfant; elle voulait la paix, le repos complet, dormir sans fin. (Une fille de ferme, 91-92)

Hormis la première structure qui établit une relation appositive entre «le délire » et «ce délire », l'asyndète se généralise dans cet exemple ; une construction paradigmatique s'établit entre «le délire » et «un besoin fou de partir» tandis qu'un autre paradigme étage les infinitifs caractérisants. Là encore, l'homogénéité sémantique est assurée entre les éléments juxtaposés : «fou » renvoie à « délire »; «partir, s'échapper, courir » sont reliés par l'idée de «fuite ». L'amplification rythmique accompagne la gradation sémantique lisible entre les items juxtaposés. La reprise de «fini » dans la seconde phrase est développée par un complément de sens absolu qui trouve un écho sous le versant négatif dans «sans fin ». La séquence «la paix, le repos complet, dormir sans fin», suite hétérogène sur le plan syntaxique, s'affirme comme chaîne orientée et graduelle, en se refermant sur une périphrase euphémistique qui désigne la mort, hyperbole de la paix et du repos.

Les cas qui illustreraient comme le comble de la reformulation sont ceux à rattacher à la réduplication. La reprise du même mot est répétition en apparence mais, par son inscription dans la chaîne textuelle, la seconde occurrence se charge d'un sens non complètement identique à celui de la précédente et oriente vers une lecture hyperbolique de la séquence. Hormis les cas de réduplication qui dénotent la parlure d'un personnage (notamment celle des personnages de l'Histoire d'une fille de ferme), la réduplication joue le rôle de marqueur d'intensité :

[18] Elle montait, mais doucement, doucement, et chargée d'un poids considérable. (Sur l'eau, 78)

[19] Jusqu'au village abandonné là-bas, là-bas, vers le nord. (Histoire d'une fille de ferme, 82)

Ces constructions graduelles que révèle l'asyndète confèrent une dynamique à la linéarité discursive en reflétant la parole en action, qui procède par ajouts et ajustements successifs. À l'échelle de la phrase, l'asyndète joue un rôle structurel. Si on admet que «le texte se présente comme une extrapolation de la phrase $»^{10}$, on peut se demander si l'asyndète joue un rôle du même ordre à l'échelle du texte.

\footnotetext{
${ }^{9}$ « Il avait sept ou huit ans. Il était un peu pâlot, très propre, avec l'air timide, presque gauche ». (Le Papa de Simon, 133). La même tension est pressentie entre «timide» et «gauche» dans cette phrase qui se referme encore sur un syntagme modifié par l'adverbe « presque », de clôture.
}

${ }^{10}$ M. Wilmet (1997). $§ 721$, p. 582. 
2. La parataxe asyndétique comme motif de structuration

\subsection{L'addition}

Dans les séquences descriptives, l'asyndète permet de juxtaposer différents éléments qui composent une scène ou l'esquisse d'un personnage. L'ouverture de la nouvelle En famille est à ce titre exemplaire :

[20] C'étaient de grosses dames aux toilettes farces [...] ; des messieurs las du bureau, la figure jaunie, la taille tournée, une épaule un peu remontée [...]. Leurs faces inquiètes et tristes disaient encore les soucis domestiques, les incessants besoins d'argent, les anciennes espérances définitivement déçues $[\ldots]$.

Tout près de la portière, un homme petit et gros, la figure bouffie, le ventre tombant entre ses jambes ouvertes, tout habillé de noir et décoré, causait avec un grand maigre d'aspect débraillé, vêtu de coutil blanc très sale et coiffé d'un vieux panama. (En famille, 102-103)

La simple addition d'éléments suffit à construire la séquence descriptive qui adopte un point de vue externe.

Les séquences asyndétiques se font écho quelquefois dans une suprastructure binaire contrastive qui condense le sens de la nouvelle.

\subsection{La structure en miroir}

Des nouvelles illustrent la structure globale en miroir. Deux séquences asyndétiques se répondent pour noter un retournement de situation dans Sur l'eau, encadrant l'épisode fantastique :

[21] Il faisait un temps magnifique ; la lune resplendissait, le fleuve brillait, l'air était calme et doux. (Sur l'eau, 74)

[22] Quand je rouvris les yeux, la lune était couchée, le ciel plein de nuages. L'eau clapotait lugubrement, le vent soufflait, il faisait froid, l'obscurité était profonde. (Sur l'eau, 78)

La nouvelle $A u$ printemps fait sans doute figure de parangon asyndétique. L'asyndète, en effet, affecte toutes les instances énonciatives de cette nouvelle, la voix du narrateur comme celle du personnage, narrateur-relais. Elle s'ouvre sur un paragraphe constitué d'une seule phrase qui reflète l'effervescence printanière en écho au titre de la nouvelle :

[23] Lorsque les premiers beaux jours arrivent, que la terre s'éveille et reverdit, que la tiédeur parfumée de l'air nous caresse la peau, entre dans la poitrine, semble pénétrer au cœur lui-même, il nous vient des désirs vagues de bonheurs indéfinis, des envies de courir, d'aller au hasard, de chercher aventure, de boire du printemps. (Au printemps, 156).

L'asyndète est réutilisée avec ironie par le personnage-narrateur second et pour mettre en garde le narrateur principal, cette fois sur le mode dysphorique.

[24] Mais quand revient le printemps avec ses feuilles et ses fleurs, ses brises chaudes et amollissantes, ses exhalaisons des champs qui vous apportent des troubles vagues, des attendrissements sans cause [...] Monsieur, prenez garde à l'amour ! Il est embusqué partout : il vous guette à tous les coins; toutes ses ruses sont tendues, toutes ses armes aiguisées, toutes ses perfidies préparées ! Prenez garde à l'amour !... Prenez garde à l'amour ! (158) 
Ce procédé se retrouve quelques lignes plus loin pour expliciter le syntagme martelé à trois reprises, « les saintes marques du travail » ${ }^{11}$, stigmates que porte la femme qui a mené à sa perte ce personnage, interprétées a contrario comme indices de son esprit vicié :

[25] Elles [les saintes marques du travail] veulent dire tous les commérages de l'atelier, les polissonneries chuchotées, l'esprit souillé par toutes les ordures racontées, la chasteté perdue, toute la sottise des bavardages, toute la misère des habitudes quotidiennes, toute l'étroitesse des idées propres aux femmes du commun $[\ldots](160)$

Et enfin pour décrire la faillite du couple, comme une confirmation de la désillusion consommée :

[25] Alors, elle vous injurie du matin au soir, ne comprend rien, ne sait rien, jacasse sans fin, chante à tuetête la chanson de Musette (oh ! la chanson de Musette, quelle scie !), se bat avec le charbonnier, raconte à la concierge les intimités de son ménage, confie à la bonne du voisin tous les secrets de l'alcôve, débine son mari chez les fournisseurs, et a la tête farcie d'histoires si stupides, de croyances si idiotes, d'opinions si grotesques, de préjugés si prodigieux, que je pleure de découragement, monsieur, toutes les fois que je cause avec elle. (162)

Le retour du même procédé acquiert ainsi une valeur structurelle et signifiante. Le contraste établi entre les diverses séquences favorise une vision pessimiste de la société et du monde. Se pourrait-il que l'asyndète fonctionne finalement comme exemplification syntagmatique des propriétés définitoires du genre même de la nouvelle?

3. En guise de conclusion: La parataxe asyndétique comme exemplification générique

L'asyndète propose une structuration syntaxique minimale qui progresse par empilement d'unités entre lesquelles aucun lien n'est explicité mais dont la relation est à reconstruire par inférence par le lecteur, qu'il s'agisse d'un processus d'addition ou de reformulation. Le recueil de nouvelles ne procède pas différemment. Les nouvelles se succèdent, rassemblées dans un même ouvrage, sans lien exprimé mais dont la seule réunion laisse supposer une intention porteuse de sens de même que leur contiguïté implique une interaction mutuelle qui en infléchit le sens ou la compréhension. Certes, le principe d'organisation d'un recueil quel qu'il soit repose sur un processus tabulaire de fait. Cependant, chez Maupassant et dans le corpus étudié, la récurrence du processus est telle qu'on ne peut s'empêcher d'y lire un effet de miroir avec la constitution du recueil même.

La construction paradigmatique ou tabulaire prévaut au niveau syntaxique, au niveau textuel, au niveau même du genre. La nouvelle, « expansion d'un noyau $~^{12}$, trouve son reflet dans les phrases à étagement. La composition sérielle mise sur une poétique du fragmentaire. De même que chaque unité juxtaposée à une autre contribue à la construction d'un ensemble phrastique et textuel, chaque nouvelle constitue un maillon de la chaîne du recueil. Les fragments sont autant de maillons d'une chaîne, à la fois fermés sur eux-mêmes et solidaires des autres. L'incomplétude de chacun est un appel au similaire. La pratique de la fragmentation formelle serait-elle un équivalent de l'appréhension de la totalité ? S'opposant à l'encyclopédisme, la mise en forme fragmentaire est signe de l'absence de la totalité et seule voie d'accès à cette totalité. L'asyndète révèle une vision parcellaire du monde chez Maupassant, une vision par facettes dont la solidarisation est confiée au lecteur.

\footnotetext{
${ }^{11}$ Le syntagme est à trois reprises mis en valeur par le présentatif «voici », puis par la phrase à constituant emphatique, enfin par sa place en fin de phrase, en clôture même du paragraphe.

${ }^{12}$ D. Grojnowski (1988). « Avant-propos. L’Amateur de nouvelles » in Maupassant. Miroir de la nouvelle.
} 
La citation suivante extraite de Pierre et $\mathrm{Jean}^{13}$ ne dit pas autre chose :

La vie, en outre, est composée des choses les plus différentes, les plus imprévues, les plus contraires, les plus disparates; elle est brutale, sans suite, sans chaîne, pleine de catastrophes inexplicables, illogiques et contradictoires qui doivent être classées au chapitre faits divers.

\section{Bibliographie}

Adam J.-M. (1990). Éléments de linguistique textuelle, Liège : Mardaga.

Béguelin M .-J. (2009). « Noyaux prédicatifs juxtaposés ». In M.-J. Béguelin, M. Avanzi, \& G. Corminboeuf (Eds.), La Parataxe, tome 1 (pp. 3-33). Berne: Peter Lang.

Greimas A.-J. (1976). La sémiotique du texte : exercices pratiques. Paris, Le Seuil.

Langue française (1987). La reformulation du sens dans le discours, $\mathrm{n}^{\circ} 73$.

Le Bot M.-Cl., Schuwer M., Richard É. (2008). La reformulation : marqueurs linguistiques, stratégies énonciatives, Rennes, PUR.

Steuckardt A., Niklas-Salminen A. (2005), Les marqueurs de glose, Aix-en-Provence : PUP.

Vial (1954) A. Guy de Maupassant et l'art du roman, Paris : Nizet.

Wilmet M. (1997). Grammaire critique du français, Louvain-la-Neuve, Duculot.

\footnotetext{
${ }^{13}$ Maupassant, Pierre et Jean, Paris, Garnier, 1959, p. 11.
} 